\title{
Post Resuscitation Care: Level of Awareness on Therapeutic Hypothermia/Targeted Temperature Monitoring among Healthcare Providers in Nairobi, Kenya
}

\author{
Hannah Khahugani Inyama ${ }^{1 *}$, Grace $0 \mathrm{moni}^{1}$, Matthew Gallek ${ }^{2}$ and Anne Karani ${ }^{1}$ \\ ${ }^{1}$ University of Nairobi, Kenya \\ ${ }^{2}$ University of Arizona, Kenya
}

Submission: March 24, 2017; Published: May 25, 2017

*Corresponding author: Hannah Khahugani Inyama, University of Nairobi, Kenya, Tel: 254-723- 065246; 254-20-2711250;

Email: hannahinyama@gmail.com

\begin{abstract}
Background: Patients who suffer cardiac arrest are susceptible to cerebral hypoxia and ischemia that leads to poor neurological outcomes in and out of hospital settings. Sudden cardiac arrest (SCA) is a leading cause of death in the USA and Canada [1]. Cardiac arrest patients', who have return of spontaneous circulation (ROSC), may have poor functional outcomes as a result of hypoxic and ischemic insults sustained during and after the arrest period [2]. The neurological insults are largely dependent on the efficiency and quality of post resuscitation care offered by the health care providers [3]. For more than five decades now, the prognosis of sudden cardiac arrest has changed for the better in first world countries following the adoption of therapeutic hypothermia/ targeted temperature management (TH/TTM) during post resuscitation care. Locally, the situation is different. Critical Care Units (CCUs) at Kenyatta National and referral hospital (the largest public hospital in Kenya) has a mortality rate of $45 \%$ following cardiac arrest (KNH Monthly mortality minutes, 2010). Anecdotal evidence shows that TH/TTM has to some extent, been embraced locally, more so in the private hospitals following the adoption of recommendations from American Heart Association, ILCOR as well as the continuous professional development training programs offered, such as, Advanced Cardiac Life Support (ACLS). The investigator sought to find out the level of awareness on TH/TTM post cardiac arrest, among health care providers working in Kenya's largest public referral hospital in Nairobi.
\end{abstract}

Objective: To determine the level of awareness of therapeutic hypothermia/ TTM among healthcare providers at Kenyatta National Hospital's (KNH), CCU.

Methods: This was a cross sectional descriptive study conducted at the KNH's 22 bed- Main CCU. Once ethical clearance was obtained, healthcare providers who worked in the CCU were purposively selected. A total of 54 participants consented to participation.

Results: The investigator revealed that, a majority of the health care providers who participated in the study were critical care nurses $(\mathrm{n}=38,71 \%)$. Most of the health care providers' years of working experience in the CCU was between 1-5 years $(\mathrm{n}=23,43 \%)$. A majority of the participants were aware of TH/TTM 35(65\%) however the healthcare providers designation did not translate to awareness level of TH/TTM ( $p=0.211,95 \% \mathrm{CI}$ ). All participants affirmed that TH/TTM was not being practiced in KNH-CCU, there was no protocol on TH/TTM and that there was need for it to be introduced in the hospital's CCUs ( $<<0.001,95 \% \mathrm{CI})$. The investigator concluded that, the participants were aware of TH/ TTM in the management of patients post cardiac arrest however this did not translate into clinical practice for all the healthcare providers in this study. The recommendations therefore are; strengthening the current level of awareness and practice through Continuous Professional Development (CPD) among health care providers on TH/ TTM and, development of TH/ TTM protocol at the institution for it's availability and use can help in increasing the familiarity levels of TH/ TTM and optimize post resuscitation care in patients following cardiac arrest.

Keywords: Therapeutic hypothermia; Targeted temperature monitoring; Cardiac arrest; Health care providers; Post resuscitation care

\section{Introduction and Background Information}

The goal of resuscitation is to support and restore effective oxygenation, ventilation and circulation with return of intact neurologic function [4]. Effective post-cardiac arrest care consists of identification and treatment of the precipitating cause of cardiac arrest combined with the assessment and mitigation of ischemia-reperfusion injury to multiple organ systems. Good 
patient neurological outcomes are majorly dependent on the quality of care provided by the health care providers during and after post cardiac arrest [3].

One of the treatment modalities currently in use for these patients population is therapeutic hypothermia/ Targeted temperature management (TTM). Therapeutic hypothermia is a treatment modality that has been in existence since the 1950s. It has been successfully used in patients following cardiac arrest, to protect the brain from global hypoxic and ischemic damages. Since then, induction of hypothermia after ROSC has been associated with improved functional recovery and reduced neurological deficits in patients of cardiac arrest $[2,5,6]$.

\section{Literature Review}

High quality post resuscitation care is crucial for the immediate and long term positive patient outcomes post cardiac arrest. The health care providers managing patients post cardiac arrest have to be equipped with the knowledge and skills to effectively manage this vulnerable population.

Several studies have been done to demonstrate awareness level and practice of TH/TTM on patients post cardiac arrest, to try and explain the uptake levels of this therapy. A study done by Toma A et al. [7], identified lack of familiarity, availability of concrete therapeutic hypothermia protocols and process issues for example, lack of availability of equipment, equipment costs, and high workload demands for emergency nurses, variable nursing awareness and variable staff uptake were some of the factor contributing to the varied uptake of TH/TTM.

Similarly, Abella [8] revealed that, despite compelling data supporting use TTM, hypothermia has yet to be broadly incorporated into physician practice. The study highlighted the need for improved awareness and education regarding this treatment option.

Moreover, Kozik [3] in her study concluded that, the use of the $\mathrm{TH} / \mathrm{TTM}$ protocol had caused a significant increase in awareness of the TTM/ TH amongst staff members in the hospital.

\section{Results}

\section{Characterization of the health care providers in CCU}

A majority of the respondents were critical care nurses $(\mathrm{n}=38,71 \%$ ) working in the CCU, followed by the medical officers $(n=7,13 \%)$. Most of the participants were aged between 31-39 years $(n=20,37 \%)$. This is illustrated in Table 1.

Table 1: Characterization of health care providers in the CCU.

\begin{tabular}{|c|c|c|}
\hline Baseline Characteristic & Freq(n) & Percent (\%) \\
\hline Age (years) & & \\
\hline$<30$ & 15 & 27.8 \\
\hline $31-39$ & 20 & 37 \\
\hline $40-49$ & 15 & 28.8 \\
\hline
\end{tabular}

\begin{tabular}{|c|c|c|}
\hline $50+$ & 4 & 7.4 \\
\hline Gender & & \\
\hline Male & 15 & 27.8 \\
\hline Female & 39 & 72.2 \\
\hline Designation & & \\
\hline Consultant & 3 & 5.6 \\
\hline Registrar & 2 & 3.7 \\
\hline SHO (Medical officer) & 7 & 13 \\
\hline Intern (Doctor) & 2 & 3.7 \\
\hline Senior Nursing Officer & 18 & 33 \\
\hline Nursing Officer (I-III) & 20 & 38 \\
\hline Total & 54 & 100 \\
\hline
\end{tabular}

Mean age $=35.9$, Median $=35.0$, Mode $=35, \mathrm{SD}=6.84$, Range $=30$ There was a positive correlation coefficient, $r(0.336)$ between gender and designation (95\% Cl; 0.06- 0.09; $\mathrm{p}<0.013)$.

\section{Health care providers' working experience in CCU}

Most of the participants had 1-5 years $(n=23,43 \%)$ work experience in CCU setup as illustrated in Figure 1.

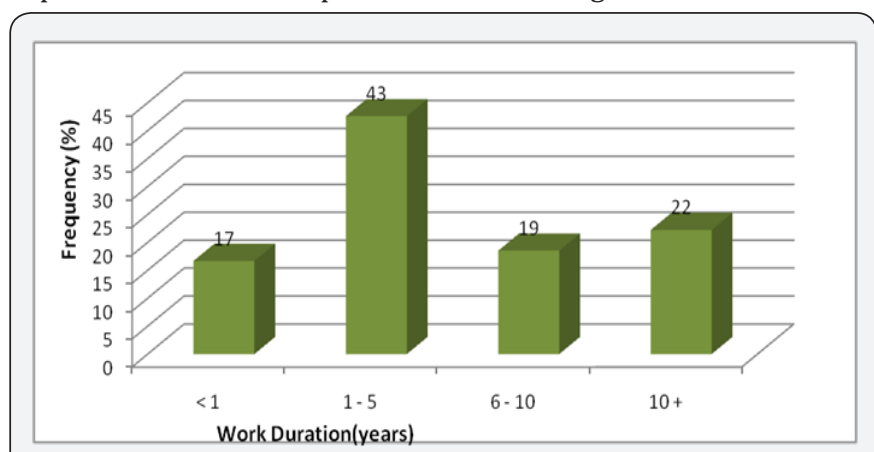

Figure 1: Health care providers' Working experience in CCU.

There was a positive correlation coefficient, $r(.472)$ linear relationship between health care providers' age and years worked in CCU $(p<$ .001).

\section{Average number of cardiac arrests in CCUs in a month}

A majority of the participants $(n=28,52 \%)$ indicated that there were more than 10 cardiac arrests occurring in the CCU within a month as summarized in Figure 2.

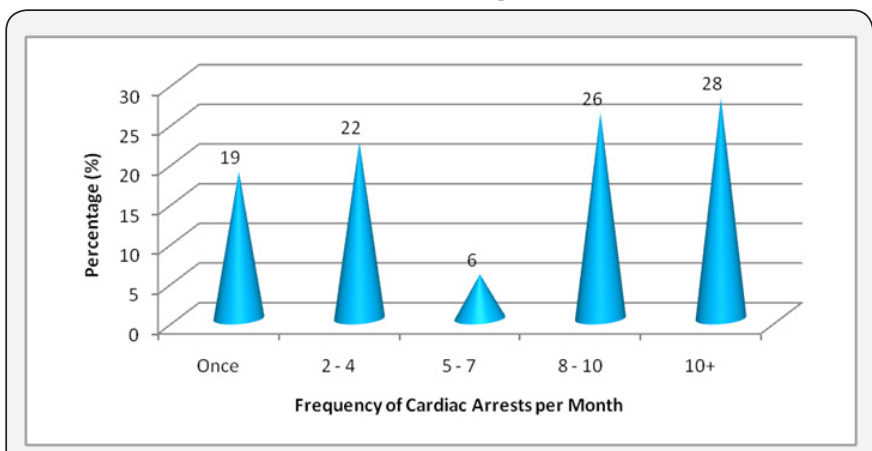

Figure 2: Average number of cardiac arrests/ month in CCU. 


\section{Participation in cardiac arrest}

With the exception of one participant, the rest 53 (98\%) had participated in the resuscitation of patients post cardiac arrest. This is illustrated in Figure 3.

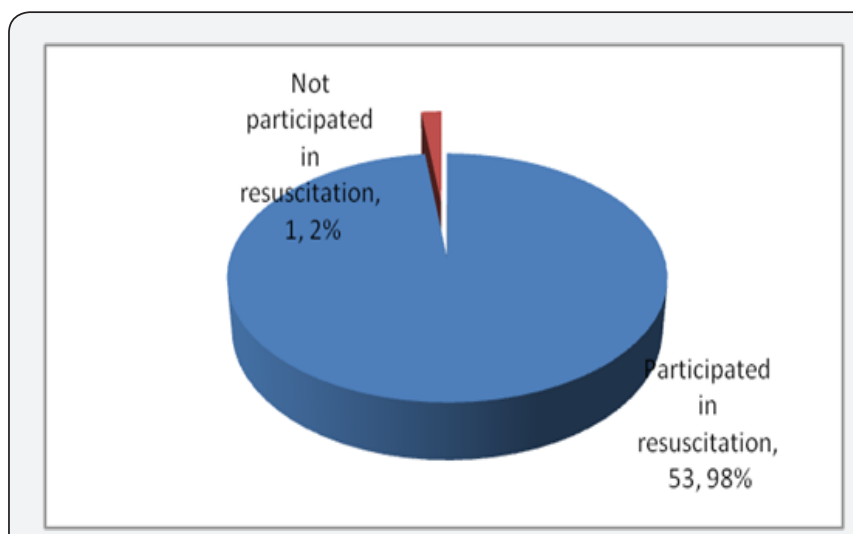

Figure 3: Health Care Providers' Participation in Cardiac arrest.

\section{Basic life support and advanced cardiac life support (BLS \&ACLS) trained}

The participants were asked whether they were BLS and ACLS trained and certified in the recent two years. All participants 54 $(100 \%)$ indicated that they were ACLS trained and certified, using the American Heart Association (AHA) testing tools for, 2011 guidelines.

\section{Awareness of therapeutic hypothermia/ targeted temperature management}

The participants were asked if they have ever heard of the therapy therapeutic hypothermia. As illustrated in Figure 4, a majority 35(65\%) had heard about it and only 19 (35\%) never heard about it.

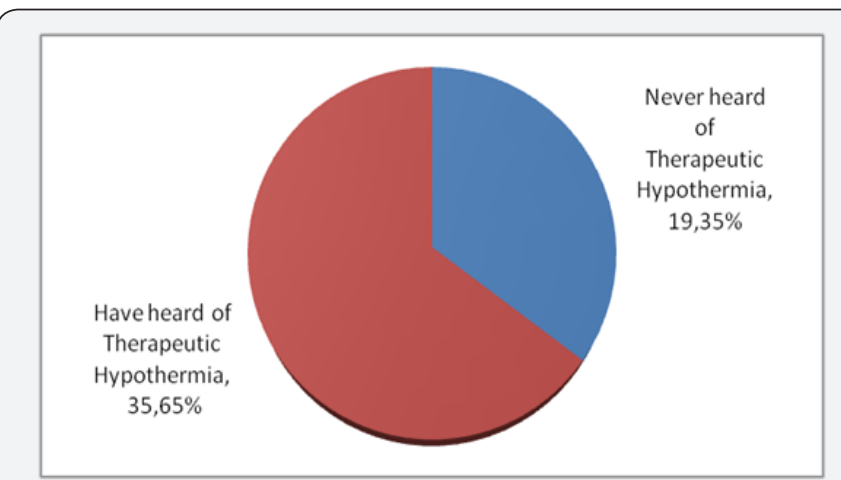

Figure 4: Awareness of TH/TTM by health care providers in CCU.

\section{Goal of inducing TH/ TTM}

Among the participants who had heard about therapeutic hypothermia, a majority $(n=21,61 \%)$ correctly indicated that it involved lowering of body temperature and subsequently the body's, cerebral metabolism whereas 14 (39\%), gave an incorrect response.

\section{Association between the health care providers' designation and awareness of TH/TTM}

Most of the participants were aware of TH/TTM 35(65\%) however there was no association, that is, the health care providers designation did not translate to awareness of TH/TTM [p=0.211, 95\%CI]. This is illustrated in Table 2 .

Table 2: Relationship between designation and knowledge of $\mathrm{TH} /$ TTM.

\begin{tabular}{|c|c|c|c|c|c|}
\hline \multirow{2}{*}{ Ever Heard of the TH/TTM } & \multicolumn{2}{|c|}{ Yes } & \multicolumn{2}{|c|}{ No } & \multirow[t]{2}{*}{ P Value } \\
\hline & Freq. & $\%$ & Freq. & $\%$ & \\
\hline \multicolumn{6}{|l|}{ Designation } \\
\hline BscN & 1 & 50 & 1 & 50 & \\
\hline Consultant & 30 & 100 & 0 & 0 & \\
\hline Intern & 1 & 100 & 0 & 100 & \\
\hline KRCHN & 2 & 66.7 & 1 & 33.3 & 0.211 \\
\hline NO I & 6 & 60 & 4 & 40 & \\
\hline NO II & 5 & 100 & 0 & 0 & \\
\hline Registrar & 1 & 33.3 & 2 & 66.7 & \\
\hline SHO & 6 & 66.7 & 3 & 33.3 & \\
\hline SNO & 13 & 72.2 & 5 & 27.8 & \\
\hline Total & 35 & 65 & 19 & 35 & \\
\hline $\begin{array}{l}\text { TH/ TTM is currently } \\
\text { Practiced in your CCUs }\end{array}$ & 0 & 0 & 54 & 100 & \\
\hline $\begin{array}{c}\text { Approved institutional } \\
\text { protocol on TH/TTM in the } \\
\text { CCU }\end{array}$ & 0 & 0 & 54 & 100 & \\
\hline $\begin{array}{l}\text { Need to have TH/TTM } \\
\text { introduced in CCUs }\end{array}$ & 54 & 100 & 0 & 0 & $<0.001$ \\
\hline
\end{tabular}

All participants affirmed that TH/TTM was not being practiced in $\mathrm{KNH}$ $\mathrm{CCU}$, there was no protocol on TH/TTM and that there was need for it to be introduced in the hospital's CCUs. $(\mathrm{P}<0.001,95 \% \mathrm{Cl})$.

\section{Discussion}

There were disparities in the level of awareness of $\mathrm{TH} /$ TTM among the health care providers in the KNH, CCU and the awareness level did not translate into practice. This cut across all designations practicing in the unit. These findings are similar to those in the study by Abella [8] and Toma et al. [7] that demonstrated that health care providers, physicians to be specific, lacked the knowledge on TH/TTM hence did not apply it in practice. They documented that lack of incorporation of the TH/TTM protocol into ACLS training guidelines exacerbated the lack of knowledge.

In this study, the participants indicated that there was no institutional protocol in place and that they would have wanted TH/TTM practice at the institution. These findings concur with Kozik T [3], who determined that, the lack of use of the protocol is a contributor to reduced levels of awareness/ familiarity.

All the health care providers who participated in this study were BLS and ACLS trained and certified. This could explain the 
reason as to why the awareness level was high in comparison to practice [9].

\section{Conclusion}

The researcher therefore concludes that:

A. Health care providers were up to date on BLS and ACLS.

B. The health care providers were aware of TH/ TTM.

C. There was no Institutional TH/ TTM protocol.

\section{Recommendations}

The researcher therefore recommends that:

A. Strengthening the current level of awareness and practice through Continuous Professional Development (CPD) among health care providers on TH/ TTM.

B. Development of TH/ TTM protocol at the institution and it's use can help in increasing the familiarity levels.

C. Further research to be conducted in other health care provision facilities, more so, in the private sector where $\mathrm{TH} /$ TTM practice is prominent for a more generalizable picture.

\section{References}

1. Urden L, Stacy K, Lough M (2010) Critical Care Nursing Diagnosis and Management. ( $6^{\text {th }}$ edn), Mosby Elsevier, USA, p. 757.
2. Nolan JP, Morley PT, Hoek V, Hickey RW, Kloeck WGJ, et al. (2003) Therapeutic Hypothermia After Cardiac Arrest. In Circulation Journal 108: $118-121$

3. Kozik TM (2007) Induced Hypothermia for Patients With Cardiac Arrest; Role of a Clinical Nurse Specialist. Crit care nurse 27(5): 36-42.

4. AHA (2011) Advanced Cardiovascular Life Support Provider Manual. AHA Printing, India, p. 45.

5. Tran PB, McGuire CV, Maloney MA (2010) Use Of Mild Therapeutic Hypothermia Improves Outcomes In Cardiac Arrest. JAAPA 23(3): 4348.

6. Bro-Jeppesen J, Kjaerkaard J, Horsted T, Wanscher MC, Nielsen SL, et al. (2010) The impact of therapeutic hypothermia on neurological function and quality of life after cardiac arrest. Resuscitation 80(2): 171-176.

7. Toma A, Bensimon CM, Dainty KN, Rubenfeld GD, Morrison LJ, et al. (2010) Perceived Barriers To Therapeutic Hypothermia For Patients Resuscitated From Cardiac Arrest: A Qualitative Study Of Emergency Department And Critical Care Workers. Crit Care Med 38(2): 504-509.

8. Abella BS, Rhee JW, Huang K, Vanden Hoek TL, Becker LB (2011) Induced Hypothermia Is Underused From Cardiac Arrest: Current Practice Survey. Resuscitation 64(2): 181-186.

9. Schefold JC, Storm C, Kruger A, Ploner CJ, Hasper D (2009) The Glascow Coma Scale is a predictor of good outcome in cardiac arrest patients treated with therapeutic hypothermia. Resuscitation 80(6): 658-661.

\begin{tabular}{l} 
Your next submission with Juniper Publishers \\
will reach you the below assets \\
- Quality Editorial service \\
- Swift Peer Review \\
- Reprints availability \\
- E-prints Service \\
- Manuscript Podcast for convenient understanding \\
- Global attainment for your research \\
- Manuscript accessibility in different formats \\
( Pdf, E-pub, Full Text, Audio) \\
- Unceasing customer service \\
Track the below URL for one-step submission \\
https://juniperpublishers.com/online-submission.php \\
\hline
\end{tabular}

